\title{
Prevalência de displasia do desenvolvimento do quadril em uma maternidade de São Paulo, Brasil ${ }^{*}$
}

\section{Prevalence of Developmental Dysplasia of the Hip in a Maternity Hospital in São Paulo, Brazil}

\author{
Giovanna Galvão Braga Motta ${ }^{1,20}$ Alessandra Rodrigues Silva Chiovatto ${ }^{20}$ \\ Eduardo Davino Chiovatto ${ }^{20}$ Márcio Luís Duarte ${ }^{10}$ Natasha Vogel Majewski Rodrigues ${ }^{30}$ \\ Wagner lared ${ }^{1,2}$
}

\footnotetext{
1 Universidade Federal de São Paulo, Departamento de Saúde Baseada em Evidências, São Paulo, São Paulo, Brasil

${ }^{2}$ Centro de Aperfeiçoamento e Pesquisa em Ultrassonografia Prof. Dr. Giovanni Guido Cerri (DASA), Departamento de Ultrassonografia São Paulo, São Paulo, Brasil

${ }^{3}$ Hospital do Servidor Público Municipal, Departamento de Ortopedia pediátrica, São Paulo, São Paulo, Brasil
}

\author{
Endereço para correspondência Márcio Luís Duarte, MD, Rua \\ Napoleão de Barros, 865, São Paulo, SP - 04024-002, Brasil \\ (e-mail: marcioluisduarte@gmail.com).
}

Rev Bras Ortop 2021;56(5):664-670.

\begin{abstract}
Resumo
Objetivo Avaliar a prevalência de displasia de desenvolvimento de quadril (DDQ), ou seja, quadris de tipo de Graf Ilc ou maior, em amostra da população de recém-nascidos de 0 a 3 dias de vida, e correlacionar os achados com os principais fatores de risco descritos na literatura.

Métodos Estudo observacional, transversal e prospectivo em amostra de recémnascidos, em um hospital maternidade em São Paulo, para avaliar a prevalência da DDQ diagnosticada pelo método de Graf e verificar sua correlação com os fatores de risco. Resultados Um total de 678 recém-nascidos foram submetidos a ultrassonografia de quadril (1.356 quadris). A prevalência de DDQ (quadris tipo Ilc, D, IIla, IIIb, e IV de Graf) foi de 5,46\%. A análise de regressão logística mostrou razões de chances (RCs) com significância estatística para os parâmetros etnia branca $(R C=2.561$; intervalo de confiança de $95 \%$ [IC95\%]: 1,07 a 6,11), multiparidade ( $R C=3,50$; IC95\%: 1,62 a 7,38$)$, sexo feminino

Palavras-chave

- luxação congênita de quadril

- ultrassonografia

- diagnóstico

- recém-nascido ( $R C=4,95$; IC95\%: 1,86 a 13,13), e apresentação pélvica $(R C=2,03$; IC95\%: 1,01 a 4,11). Conclusão A prevalência de DDQ na amostra foi de $5,45 \%$ usando a ultrassonografia como método de diagnóstico. Este resultado é diferente do dos estudos que avaliam a prevalência exclusivamente do exame físico (manobra de Ortolani). Os principais fatores de risco associados ao maior risco de DDQ foram recém-nascidos do sexo feminino, com apresentação pélvica, primogênitos, e de etnia branca.
\end{abstract}

Trabalho desenvolvido no Hospital e Maternidade Escola Dr. Mário de Moraes Altenfelder Silva, São Paulo, São Paulo, Brasil.

recebido

30 de Outubro de 2020

aceito

25 de Junho de 2021
DOI https://doi.org/ $10.1055 / \mathrm{s}-0041-1736407$ ISSN 0102-3616.
(C) 2021. Sociedade Brasileira de Ortopedia e Traumatologia. All rights reserved.

This is an open access article published by Thieme under the terms of the Creative Commons Attribution-NonDerivative-NonCommercial-License, permitting copying and reproduction so long as the original work is given appropriate credit. Contents may not be used for commercial purposes, or adapted, remixed, transformed or built upon. (https://creativecommons.org/ licenses/by-nc-nd/4.0/)

Thieme Revinter Publicações Ltda., Rua do Matoso 170, Rio de Janeiro, RJ, CEP 20270-135, Brazil 
Abstract
Keywords
- hip dislocation, congenital
- ultrasonography
- diagnosis
- newborn

Objective To evaluate the prevalence of developmental dysplasia of the hip (DDH), that is, hips classified as Graf type-Ilc or higher, among a sample of the population of newborns aged from 0 to 3 days of life, and to correlate the findings with the main risk factors described in the literature.

Methods An observational, cross-sectional, prospective study on a sample of newborns at a Maternity Hospital School in the city of São Paulo, Brazil, to assess the prevalence of DDH diagnosed by the Graf method and verify its correlation with the risk factors.

Results A total of 678 newborns underwent hip ultrasound (1,356 hips). The prevalence of DDH was of $5.46 \%$. The logistic regression analysis showed odds ratios (ORs) with statistical significance for the following parameters: white ethnicity (OR $=2.561 ; 95 \%$ confidence interval $[95 \% \mathrm{Cl}]: 1.07$ to 6.11$)$; multiparity $(\mathrm{OR}=3.50 ; 95 \% \mathrm{Cl}$ : 1.62 to 7.38 ), female gender ( $\mathrm{OR}=4.95 ; 95 \% \mathrm{Cl}: 1.86$ to 13.13 ); and breech presentation (OR=2.03; $95 \% \mathrm{Cl}: 1,01$ to 4.11 ).

Conclusion The prevalence of DDH in the sample was of $5.45 \%$ using ultrasound as a diagnostic method. This result is different from that of studies that assessed prevalence exclusively through physical examination (Ortolani maneuver). The main risk factors associated with a higher risk of developing DDH were newborns of the female gender, with breech presentation, firstborns, and of white ethnicity.

\section{Introdução}

Faltam estudos e evidências disponíveis para orientar a prática clínica no tratamento da displasia de desenvolvimento do quadril (DDQ). Entre as limitações observadas, não há consenso quanto à classificação e o diagnóstico da DDQ e uma ampla variabilidade na tomada de decisão, quase sempre orientada por estudos com amostras pequenas. ${ }^{1}$ A maioria dos estudos existentes sobre DDQ são retrospectivos, têm amostras pequenas (considerandose o número de indivíduos), e não corrigem os resultados quando os casos de DDQ bilateral são incluídos. ${ }^{1}$ Muitos estudos também não incluem todo o espectro da DDQ. ${ }^{1}$

No estudo publicado por Guarniero et al. ${ }^{2}$ (1988) com base em uma metanálise e protocolos de regressão logística múltipla, a prevalência estimada dessa condição no Brasil foi próxima de $1,1 \%$, e esse dado foi obtido por meio da positividade da manobra de Ortolani. Para melhorar as evidências disponíveis, é necessário utilizar uma estrutura padronizada para relatórios e diagnósticos, com consistência, bem como realizar estudos prospectivos com um desenho metodológico sólido. ${ }^{1}$

A displasia acetabular não pode ser excluída por um exame físico normal, e o ultrassom pode ser uma ferramenta importante para o diagnóstico precoce e tratamento da doença. A instabilidade articular e a luxação do quadril podem ser diagnosticadas por exame físico usando as manobras de Barlow e Ortolani, respectivamente. ${ }^{3}$

Este estudo tem como objetivo avaliar a prevalência da DDQ em recém-nascidos de 0 a 3 dias de vida em uma maternidade pública, e correlacionar os achados com os principais fatores de risco descritos na literatura.

\section{Materiais e Métodos}

Este estudo foi aprovado pelo Comitê de Ética das duas instituições envolvidas, sob os números 1.554 e 2.016. Este artigo foi escrito seguindo as diretrizes da iniciativa Strengthening the Reporting of Observational Studies in Epidemiology (STROBE) para a comunicação de estudos observacionais. $^{4}$

Este estudo observacional, transversal, e prospectivo foi realizado em maternidade pública de alto risco da cidade de São Paulo com uma média de 7.900 partos por ano. Realizamos os exames no berçário porque essa seria a única vez que teríamos uma amostra minimamente aceitável para avaliar a prevalência na população de nascidos vivos, embora a melhora espontânea na classificação tenha sido descrita na literatura, especificamente nos quadris que não apresentam instabilidade no exame dinâmico. A coleta foi realizada aleatoriamente quando a pesquisadora principal e sua equipe compareceram ao hospital, de março a setembro de 2018. Os pais ou responsáveis legais foram informados sobre as condições do estudo por meio do Termo de Consentimento Livre e Esclarecido, apresentado antes do exame de ultrassonografia.

A população deste estudo epidemiológico consistiu em recém-nascidos internados de 0 a 3 dias de vida para a detecção precoce da doença. Cada participante foi avaliado apenas uma vez, e frealizou-se uma amostragem aleatória. Para calcular o tamanho amostral necessário, estimou-se o número de 500 casos para se obter uma incidência de até $5,5 \%$, com uma taxa de erro estimada de até $2 \%$. Estabeleceuse um intervalo de confiança de 95\% (IC95\%). Estimou-se um aumento de $30 \%$ no tamanho da amostra, uma vez que, após a coleta, as avaliações dos fatores de covariança e de risco seriam realizadas por meio da análise multivariada, com 
possível perda de dados. Assim, o tamanho amostral necessário para este estudo foi estimado em pelo menos 650 casos.

0 critério de inclusão foi recém-nascidos internados na maternidade nas datas em que a equipe de pesquisa atendeu ao local.

Os critérios de exclusão foram:

1) Parto prematuro extremo: devido à necessidade de oxigênio e aquecimento na incubadora, e para evitar perda excessiva de calor e a manipulação do recémnascido em risco, pois é necessário usar gel frio e mudar a posição do recém-nascido para realizar a ultrassonografia, o que aumenta o risco de hemorragia intracraniana;

2) Dificuldade respiratória: devido à necessidade de oxigênio e monitoramento, pois é necessário manipular o recém-nascido e mudar sua posição para realizar a ultrassonografia;

3) Patologias e/ou condições que necessitassem de cuidados intensivos: para evitar manipular o recémnascido, pois é necessário mudar sua posição para realizar a ultrassonografia;

4) Anomalias congênitas, doenças genéticas: porque pacientes com doenças genéticas e/ou distúrbios congênitos têm maior prevalência conhecida de DDQ, que poderia ser avaliada como viés de seleção e aumento da prevalência, não traduzindo a displasia média na população geral;

5) Gravidez de alto risco: devido à necessidade de acompanhamento do recém-nascido;

6) Pais ou responsáveis legais que não assinaram o termo de consentimento, pois não concordaram em participar do estudo; e

7) Os quadris classificados como tipo Ila pelo método de Graf, por causa da imaturidade que poderia se normalizar em poucas semanas.

Após a aceitação para participar do estudo, realizou-se um exame de ultrassonografia de quadril nos pacientes que já haviam sido submetidos a exame clínico por um neonatologista, com as manobras de Ortolani e Barlow. ${ }^{5}$ Por fim, aplicou-se um questionário padronizado sobre o tema, elaborado pelos autores, para registrar os fatores de risco para DDQ.

\section{Exame de ultrassonografia}

O método utilizado foi desenvolvido por $\mathrm{Graf}^{6}$ em 1980; é considerado o método de referência, e é aceito por sistemas de saúde em diversos países. Todos os exames deste estudo foram realizados estritamente seguindo o método Graf.

Um médico ultrassonografista pediátrico com mais de vinte anos de experiência em ultrassonografia pediátrica de quadril, cego quanto aos resultados dos exames clínicos, realizou os exames de ultrassom.

\section{Posicionamento}

O recém-nascido é posicionado em decúbito lateral, oposto ao quadril a ser examinado, com a perna ligeiramente flexionada e aduzida, e o pé em leve rotação interna

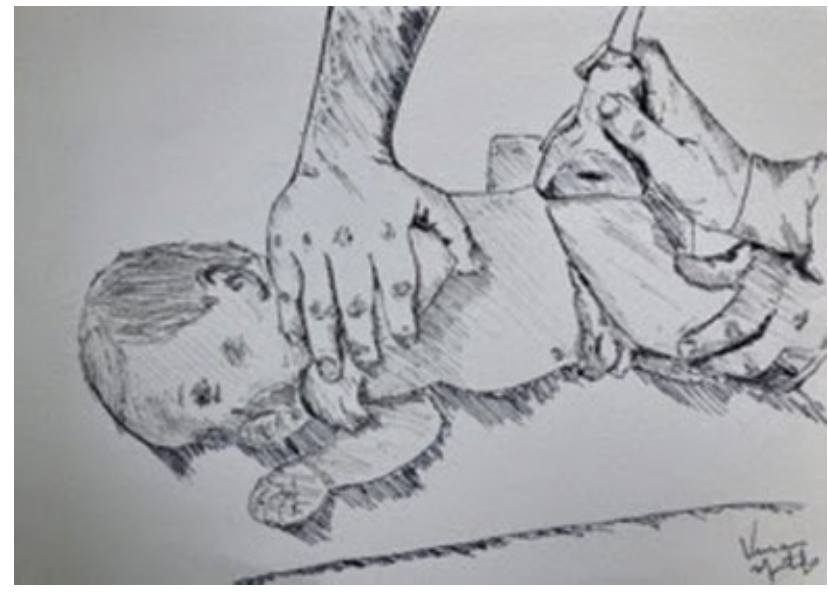

Fig. 1 Posição correta do recém-nascido durante o exame: decúbito lateral com os membros inferiores flexionados e aduzidos. Crédito: Arte de Vinicius Mustafa.

(-Fig. 1); utilizando-se um transdutor linear, no plano coronal do quadril, estabelece-se um plano padrão no qual é possível avaliar a morfologia acetabular e o grau de cobertura da cabeça femoral.

No método de Graf, o tipo I é normal, com um ângulo $\alpha>60^{\circ}$. Os quadris tipo Ila representam imaturidade, com um ângulo $\alpha$ entre $50^{\circ}$ e $59^{\circ}$, e um ângulo $\beta>55^{\circ}$. Os quadris com um ângulo $\alpha \leq 49^{\circ}$ são definidos como tendo um desenvolvimento patológico, e são classificados como tipos IIc, IId, IIIa, ou IIIb. Os quadris tipo IV não são mensuráveis. A DDQ foi definida por Graf em casos em que a classificação da ultrassonografia revela um padrão $\geq$ Iib. Os quadris Ila são classificados como imaturos, mas o acompanhamento e o tratamento são sugeridos quando persistem após 30 dias de vida. $^{7}$

\section{Análise estatística}

Os dados foram inseridos em uma planilha do programa Excel (Microsoft Corp., Redmond, WA, Estados Unidos). Para a análise estatística, os dados foram exportados para o programa Statistical Package for the Social Sciences (IBM SPSS Statistics for Macintosh, IBM Corp., Armonk, NY, Estados Unidos), versão 24.0. As estatísticas descritivas dos dados categóricos foram apresentadas de acordo com suas frequências absolutas e relativas. Os dados contínuos foram apresentados de acordo com suas médias e respectivos desvios padrão (DPs). A prevalência foi calculada pela razão entre os recém-nascidos com pelo menos um quadril com classificação $\geq$ IIc de acordo com o método de Graf e o total de recémnascidos avaliados.

Para a análise do fator de risco, realizou-se uma regressão logística binomial para verificar os efeitos do sexo (masculino e feminino), da etnia (branca e outras), da paridade (primípara ou multiparidade), e da apresentação intrauterina do feto no momento do parto (culatra ou outros) para identificar o risco aumentado de DDQ. Para a análise de regressão, foram utilizadas variáveis independentes com plausibilidade biológica que apresentaram associação após análise univariada com aceitação no modelo $p \leq 0,1$. Para que 
fosse aceito como estatisticamente significativo, na equação de previsão do fator de risco, o valor de significância devia ser $p<0,05$.

\section{Resultados}

Houve 3.970 partos entre abril e setembro de 2018, dos quais 733 recém-nascidos estavam disponíveis para exame. No entanto, nesse período, 28 recém-nascidos foram prematuros, 16 apresentaram dificuldade respiratória transitória, e 3 tiveram mecônio aspirado, sendo excluídos do estudo devido à necessidade de cuidados intensivos. Também foram excluídos oito recém-nascidos cujos pais ou responsáveis legais não aceitaram participar do estudo. Aproximadamente $8 \%$ dos recém-nascidos foram retirados da amostra de acordo com os critérios estipulados (-Fig. 2). Não houve complicações durante o exame realizado na pesquisa.

Foram incluídos 678 recém-nascidos (1.356 quadris), e a prevalência de DDQ no grupo foi de 5,46\% (37 recémnascidos). Ao todo, 24 recém-nascidos apresentavam displasia unilateral: 3 , no quadril direito (8,1\%), e 21 , no quadril esquerdo (56,7\%); e $13(35,1 \%)$ recém-nascidos tinham displasia bilateral. As características amostrais estão descritas na - Tabela 1. Entre os recém-nascidos com DDQ examinados, a prevalência foi avaliada em relação a cada tipo de quadril utilizando o método de Graf (- Tabela 2).

Entre os fatores de risco avaliados, os seguintes apresentaram significância estatística na análise univariada:

- Sexo feminino (risco relativo $[R R]=5,78$; IC95\%: 2,28 a $14,67 ; p=0,0002)$;
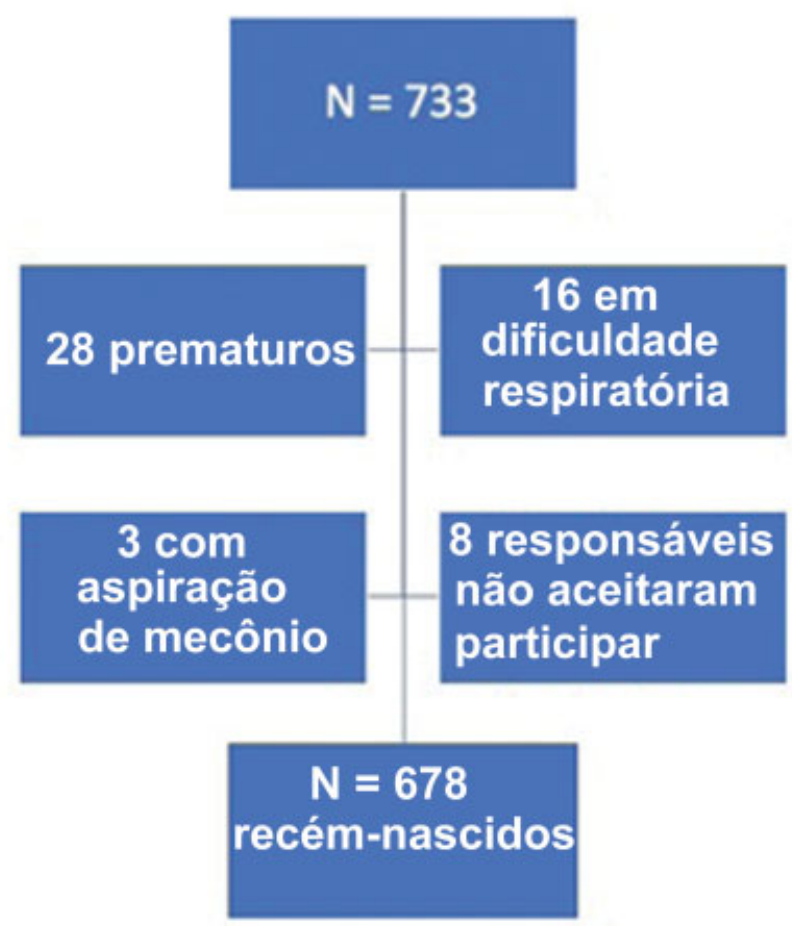

Fig. 2 Fluxograma de seleção da amostra.
- Apresentação pélvica ( $R R=2,94$; IC95\%: 1,58 a 5,48; $p=0,0007)$;

Tabela 1 Características da população estudada em relação aos fatores de risco

\begin{tabular}{|c|c|c|}
\hline Característica & $\begin{array}{l}\text { Recém-nascidos } \\
\text { avaliados } \\
(N=678)- \\
n(\%)\end{array}$ & $\begin{array}{l}\text { Recém-nascidos } \\
\text { com displasia de } \\
\text { desenvolvimento } \\
\text { do quadril } \\
(N=37 ; 5,4 \%) \text { - } \\
\mathrm{n}(\%)\end{array}$ \\
\hline \multicolumn{3}{|l|}{ Sexo } \\
\hline - Feminino & $356(52,5 \%)$ & $32(86,5 \%)$ \\
\hline - Masculino & 322 (47,5\%) & $5(13,5 \%)$ \\
\hline \multicolumn{3}{|l|}{ Etnia } \\
\hline - Branca & $364(53,7 \%)$ & $30(81 \%)$ \\
\hline - Negra & $177(26 \%)$ & 0 \\
\hline - Parda & $137(20,3 \%)$ & 7 (19\%) \\
\hline \multicolumn{3}{|l|}{ Tipo de parto } \\
\hline - Natural & $449(66 \%)$ & $22(59 \%)$ \\
\hline - Cesariana & $229(34 \%)$ & $15(41 \%)$ \\
\hline \multicolumn{3}{|l|}{ Apresentação } \\
\hline - Pélvica & $179(26,4 \%)$ & $19(51 \%)$ \\
\hline - Cefálica & $474(69,9 \%)$ & $17(46 \%)$ \\
\hline - Transversal & $25(3,7 \%)$ & $1(3 \%)$ \\
\hline \multicolumn{3}{|l|}{ Paridade } \\
\hline - Primípara & $302(44,5 \%)$ & 27 (73\%) \\
\hline - Multiparidade & $376(55,4 \%)$ & $10(27 \%)$ \\
\hline Gêmeos & $4(0,6 \%)$ & 0 \\
\hline Histórico familiar & $19(2,8 \%)$ & $1(2,7 \%)$ \\
\hline $\begin{array}{l}\text { Patologias } \\
\text { ortopédicas }\end{array}$ & 0 & 0 \\
\hline $\begin{array}{l}\text { Síndromes } \\
\text { genéticas }\end{array}$ & 0 & 0 \\
\hline $\begin{array}{l}\text { Manobra de } \\
\text { Ortolani } \\
\text { positiva }\end{array}$ & $4(0,6 \%)$ & $2(5,4 \%)$ \\
\hline
\end{tabular}

Tabela 2 Prevalência em relação a cada tipo de quadril utilizando o método de Graf $(N=1356)$

\begin{tabular}{|l|l|l|}
\hline $\begin{array}{l}\text { Classificação } \\
\text { do quadril }\end{array}$ & $\begin{array}{l}\text { Quadril direito - } \\
\text { n (\%) }\end{array}$ & $\begin{array}{l}\text { Quadril esquerdo - } \\
\text { n (\%) }\end{array}$ \\
\hline Ia & $594(43,6 \%)$ & $554(40,6 \%)$ \\
\hline Ib & $8(0,6 \%)$ & $23(2,3 \%)$ \\
\hline Ila & $60(4,4 \%)$ & $67(4,9 \%)$ \\
\hline Ilc & $1(0,07 \%)$ & $3(0,22 \%)$ \\
\hline Ild & $9(0,66 \%)$ & $23(1,65 \%)$ \\
\hline Illa & $4(0,3 \%)$ & $4(0,3 \%)$ \\
\hline Iv & $2(0,14 \%)$ & $4(0,3 \%)$ \\
\hline
\end{tabular}


- Primípara (RR=3,36; IC95\%: 1,65 a 6,83; $p=0,0008$ ); e

- Étnica branca (RR=3,69; IC95\%: 1,65 a 8,3; $p=0,001$ ).

Os fatores de risco que não apresentaram significância estatística foram:

- Cesariana ( $R R=1,33$; IC95\%: 0,70 a 2,52; $p=0,37$ );

- Histórico familiar de DDQ (RR=0,96; IC95\%: 0,14 a 6,66; $p=0,97)$; e

- Gêmeos (RR=1,80; IC95\%: 0,12 a 25,4; $p=0,66$ ).

Em relação ao histórico familiar, pode haver viés de informação devido à dificuldade de coleta de dados sobre as famílias, pois a maioria dos pais ou responsáveis legais estavam inseguros ao fornecer informações sobre o histórico familiar. Gêmeos, também considerados fator de risco, não apresentaram significância estatística. É necessário considerar um possível viés de seleção devido à prematuridade e à indicação de internação, o que foi considerado fator de exclusão neste estudo.

A sensibilidade da manobra de Ortolani para quadris com displasia (Graf $\geq$ tipo Iic) foi de apenas 5,41\% (IC95\%: 0,66\% a 18,2\%), com especificidade de 99,7\% (IC95\%: 98,8\% a 99,9\%). Para o diagnóstico de quadril deslocado (Graf tipo IV), a sensibilidade da manobra de Ortolani foi de 50\% (IC95\%: 6,76\% a 93,24\%), com especificidade de 99,7\% (IC95\%: $98,8 \%$ a $99,9 \%$ ). O valor preditivo positivo foi de $50 \%$ (IC95\%: $15,5 \%$ a $84,5 \%$ ) e o valor preditivo negativo foi de $99,7 \%$ (IC95\%: $99,2 \%$ a $99,9 \%)$.

A regressão logística binomial verificou os efeitos do sexo, da etnia, da paridade, e da apresentação do feto no momento do parto no risco aumentado de DDQ. O modelo de regressão logística foi estatisticamente significativo: $X^{2}(3)=44,553$; $\mathrm{p}<0,001$. O modelo mostrou-se capaz de explicar $18,4 \%\left(\mathrm{R}^{2}\right.$ de Nagelkerke) da variação de risco de DDQ e classificou corretamente $94,5 \%$ dos casos. Todas as quatro variáveis preditivas foram estatisticamente significativas: sexo, etnia, paridade e apresentação pélvica. Ser branco, do sexo feminino, primogênito, e ter uma apresentação pélvica aumentou o risco de DDQ como mostra a - Tabela 3.

A análise de regressão logística mostrou aumento da razão de chances (RC) para a etnia branca (OR: 2.561; IC95\%: 1,07 a 6,11), a multiparidade ( $O R=3,50$; IC95\%: 1,62 a 7,38), o sexo feminino ( $\mathrm{OR}=4,95$; IC95\%: 1,86 a
13,13), e a apresentação pélvica (OR =2,03; IC95\%: 1,01 a $4,11)$.

\section{Discussão}

A revisão bibliográfica não revelou outro estudo ultrassonográfico prospectivo que estimasse a prevalência de DDQ na cidade de São Paulo.

A DDQ pode se manifestar de 3 maneiras: pela instabilidade articular, que pode ser diagnosticada pela manobra de Barlow; por uma luxação do quadril, que pode ser diagnosticada pela manobra de Ortolani; e por uma displasia acetabular, que não pode ser excluída após um exame físico normal, e precisa da ajuda do ultrassom para o diagnóstico. A displasia acetabular é uma das formas mais comuns da doença, representada na classificação do método de Graf pelos tipos IIa, IIb, IIc e D; essa informação pode ser confirmada em nosso estudo. Como o diagnóstico tardio de DDQ pode causar sérios problemas na idade adulta, justifica-se o uso de ferramentas como o ultrassom para a identificação, principalmente da forma mais recorrente. ${ }^{3}$

Guarniero et al. ${ }^{2}$ avaliaram a prevalência de DDQ em seu estudo prospectivo/retrospectivo na cidade de São Paulo, por meio de exame físico, utilizando a positividade da manobra de Ortolani para seu cálculo estatístico, e encontraram resultado de $1,1 \%$. Não se pode comparar o presente estudo com o de Guarniero et al. ${ }^{2}$, uma vez que as metodologias são diferentes, e entendemos que recém-nascidos com displasia acetabular e que não têm quadril deslocado podem ter a doença, e a manobra de Ortolani pode ser negativa.

Barbosa e Albernaz ${ }^{8}$ foram os únicos autores que tentaram estimar a prevalência de DDQ no Brasil, em estudo retrospectivo realizado na Faculdade de Medicina da Universidade Católica de Pelotas. Os autores relataram muitos potenciais vieses de seleção, porque a análise foi realizada em um pequeno número de casos identificados a partir dos prontuários dos pacientes.

Na metanálise de Ortiz-Neira et al., ${ }^{9}$ a prevalência de DDQ foi de 1,9\% (20.196 casos de DDQ entre 1.065 .867 pacientes). Em 2019, Zhao et al. ${ }^{10}$ encontraram uma prevalência de $174,9 / 1.000$ no Tibete com um projeto de estudo semelhante ao nosso, realizando avaliações ecográficas e clínicas em recém-nascidos para DDQ ao longo de 1 ano, em 10 distritos

Tabela 3 Regressão logística binomial

\begin{tabular}{|l|l|l|l|l|l|l|l|l|l|}
\hline \multicolumn{2}{|l|}{ Intervalo de confiança de 95\% para EXP(B) } \\
\hline
\end{tabular}

Abreviação: B (Beta), coeficiente de regressão; S.E. (Standard Error), Erro padrão; DF (Degree of freedom), Graus de liberdade; Sig. (Significance), Significância estatística; Exp(B) (Exponência do Beta), Razão de chances (Odds Ratio). 
de diferentes altitudes. A prevalência de DDQ mostrou correlação significativa com altitudes elevadas. ${ }^{10}$

"A incidência por 1.000 nascidos vivos varia de 0,06 em africanos na África a 76,1 em indígenas americanos. Há uma variabilidade significativa na incidência em cada grupo racial por localização geográfica". ${ }^{11} \mathrm{O}$ papel da displasia acetabular e da osteoartrite do quadril adulto é complexo. ${ }^{11}$ Estudos arqueológicos demonstram que a epidemiologia da DDQ pode estar mudando. ${ }^{11}$

Em 2010, Pollet et al. ${ }^{12}$ encontraram uma prevalência de 6,6/1000 nascidos vivos em uma província do Canadá, ao passo que outros estudos descreveram uma incidência média de $1 \mathrm{a}$ 2/1.000 com base apenas no rastreamento clínico. Um estudo $^{13}$ conduzido no Reino Unido (RU) e na Irlanda encontrou uma incidência global de 6,7/1000 na Irlanda com base no rastreamento clínico com apresentação tardia. No RU, 0,34/1.000 recém-nascidos tiveram DDQ tardia (após 3 meses) com base em ultrassonografias feitas em um programa que envolveu 107.440 recém-nascidos. ${ }^{13}$ Na Noruega, Engesæter et al. $^{14}$ encontraram apresentação tardia (após 1 mês) em 0,32/1.000 recém-nascidos com base em ultrassonografias neonatais de um grande grupo com fatores de risco e/ou achados clínicos. Güler et al. ${ }^{15}$ relataram prevalência de $9,9 \%$ na Turquia.

Neste estudo, os principais fatores altamente associados ao aumento do risco de DDQ encontrados foram: sexo feminino ( $R R=5,78 ; p=0,0002)$, apresentação pélvica ( $R R$ $=2,94 ; p=0,0007)$, primogênito ( $R=3,36 ; p=0,0008)$, e etnia branca ( $R R=3,69 ; p=0,0015)$. A etnia negra é um fator protetor; na amostra deste estudo, não havia pacientes negros com DDQ. Havia apenas dois pares de gêmeos elegíveis para exame nos três primeiros dias de vida, que não apresentaram DDQ; portanto, isso não apresentou um valor estatístico considerável.

Apesar de as características histórico familiar e ser gêmeo terem se revelado fatores de risco significativos em outro estudo, ${ }^{16}$ em nosso estudo, não foram adequadamente avaliados por conta do viés de seleção, uma limitação do estudo que envolve principalmente fatores de exclusão, como recémnascidos em terapia intensiva, prematuridade, dificuldade respiratória, hipoglicemia, ou qualquer alteração clínica que exija cuidados específicos. ${ }^{16}$ Havia apenas dois pares de gêmeos elegíveis para exame nos três primeiros dias de vida, que não apresentaram $\mathrm{DDQ}$, não tendo, assim, um valor estatístico considerável. Não foi possível avaliar com precisão o histórico familiar, porque a maioria dos pais ou responsáveis legais não sabiam da existência de casos de DDQ na família, que, portanto, podem ter sido subestimados neste estudo. Outras limitações são o fato de que as ultrassonografias foram feitas somente por um ultrassonografista pediátrico, e a ausência de acompanhamento do desenvolvimento dos pacientes após o ultrassom. Ressalta-se também que o estudo foi realizado em um hospital da cidade de São Paulo, e relatouse a prevalência da doença neste estabelecimento.

O diagnóstico precoce é considerado essencial para um tratamento eficaz e um bom prognóstico. ${ }^{17}$ Considerando que há displasias leves, que, segundo estudos, melhoram espontaneamente em $\sim 90 \%$ dos casos, ${ }^{18}$ uma avaliação de ultrassonografia em todos os recém-nascidos que não apresentam fatores de risco ou sinais clínicos aos 21 dias de vida poderia revelar casos de imaturidade que se beneficiariam do tratamento conservador. Entendemos que há uma ampla discussão sobre o tratamento precoce ${ }^{19}$ em casos de imaturidade dos quadris. No entanto, sabemos que o impacto psicológico, familiar e socioeconômico parece ser superior se compararmos um tratamento realizado com uma cinta de Pavlik ao tratamento cirúrgico com imobilização fundiária de gesso. Carlile et al. ${ }^{20}$ sugerem que recém-nascidos com fatores de risco importantes ou sinais clínicos positivos devem ser submetidos ao exame até a sexta semana de vida.

Em relação à triagem da $\mathrm{DDQ}$, recomenda-se que a morfologia e a estabilidade sejam verificadas por ultrassonografia, além do exame clínico, que, semelhante a o que ocorre em outros países, poderia ser implementado em instituições brasileiras com fácil acesso a um ultrassonografista com experiência para realizar este exame. O exame físico nem sempre detecta a luxação acetabular precoce, o índice acetabular, e a subluxação. O diagnóstico tardio de luxação não evidencia que o teste físico foi malfeito. Um índice acetabular aumentado pode favorecer o escorregamento lateral da cabeça femoral para fora do acetábulo, desenvolvendo uma luxação tardia. O diagnóstico tardio de quadril subluxado ou deslocado não está necessariamente associado ao quadril neonatal subluxável ou luxável. ${ }^{3}$

Há uma alta taxa de exames clínicos falso-negativos, mesmo quando realizados por médicos experientes. ${ }^{21}$ Para avaliar se o resultado deste estudo reflete a prevalência da doença na população de São Paulo, são necessários novos estudos em outros centros e com amostras maiores.

\section{Conclusão}

Este estudo mostrou que a prevalência de exames de ultrassom que indicaram DDQ nesta amostra foi de 5,45\%. Os principais fatores de risco associados ao maior risco de DDQ foram sexo feminino, apresentação pélvica, primogênito, e etnia branca.

\section{Contribuição dos Autores}

GM: elaboração dos instrumentos de coleta de dados, coleta de dados, análises iniciais, conceitualização e elaboração do estudo, redação do manuscrito inicial, e redação e revisão do manuscrito final.

AC: revisão da literatura, e redação e revisão do manuscrito.

EC: análise e preparação dos dados para a análise estatística, e revisão do manuscrito.

NV: contribuição com a parte clínica da ortopedia pediátrica e a parte da radiologia musculoesquelética, e redação e revisão do manuscrito.

MLD: redação e revisão do manuscrito.

WI: conceitualização e elaboração do estudo, coordenação e supervisão da coleta de dados, redação do manuscrito inicial, revisão crítica do manuscrito quanto ao conteúdo intelectual importante, e redação e revisão do manuscrito final. 


\section{Suporte Financeiro}

Os autores não têm fontes de financiamento externo a declarar.

\section{Conflito de Interesses}

Os autores declaram não haver conflito de interesses.

\section{Agradecimentos}

Nossa equipe agradece aos Dr. Rafael Costa Hime, Pedro Alexandre F. Brevel, e Alexandre de Lourenço pela contribuição na realização deste estudo.

\section{Referências}

1 Schaeffer EK, Study Group I, Mulpuri K. Developmental dysplasia of the hip: addressing evidence gaps with a multicentre prospective international study. Med J Aust 2018;208(08):359-364

2 Guarniero R, Montenegro NB, Vieira PB, Peixinho M. Sinal de Ortolani: resultado do exame ortopédico em 9171 recém-nascidos na Associação Maternidade de São Paulo. Rev Bras Ortop 1988;23(05):125-128

3 Ilfeld FW, Westin GW, Makin M. Missed or developmental dislocation of the hip. Clin Orthop Relat Res 1986;(203):276-281

4 von Elm E, Altman DG, Egger M, Pocock SJ, Gøtzsche PC, Vandenbroucke JPSTROBE Initiative. The Strengthening the Reporting of Observational Studies in Epidemiology (STROBE) statement: guidelines for reporting observational studies. J Clin Epidemiol 2008 ; 61(04):344-349

5 Morrissy RT, Weinstein SL editors. Lovell and Winter's Pediatric orthopaedics. 4th ed. Philadelphia, PA: Lippincott-Raven; 1996

6 Graf R. Hip sonography: background; technique and common mistakes; results; debate and politics; challenges. Hip Int 2017;27 (03):215-219

7 Palocaren T, Rogers K, Haumont T, Grissom L, Thacker MM. High failure rate of the Pavlik harness in dislocated hips: is it bilaterality? J Pediatr Orthop 2013;33(05):530-535

8 Barbosa RO, Albernaz EP. Perfil dos lactentes diagnosticados com displasia do desenvolvimento do quadril. Rev Bras Ortop 2018;54 (05):497-502

9 Ortiz-Neira CL, Paolucci EO, Donnon T. A meta-analysis of common risk factors associated with the diagnosis of developmental dysplasia of the hip in newborns. Eur J Radiol 2012;81(03): e344-e351

10 Zhao L, Ma Q, Feng X, et al. Screening for Developmental Dysplasia of the Hip in Infants in Tibet Identifies Increased Prevalence Associated with Altitude. Med Sci Monit 2019;25:5771-5775

11 Loder RT, Skopelja EN. The epidemiology and demographics of hip dysplasia. ISRN Orthop 2011;2011:238607

12 Pollet V, Pruijs H, Sakkers R, Castelein R. Results of Pavlik harness treatment in children with dislocated hips between the age of six and twenty-four months. J Pediatr Orthop 2010;30(05):437-442

13 Elbourne D, Dezateux C, Arthur R, et al; UK Collaborative Hip Trial Group. Ultrasonography in the diagnosis and management of developmental hip dysplasia (UK Hip Trial): clinical and economic results of a multicentre randomised controlled trial. Lancet 2002; 360(9350):2009-2017

14 Engesæter IØ, Laborie LB, Lehmann TG, et al. Prevalence of radiographic findings associated with hip dysplasia in a population-based cohort of 2081 19-year-old Norwegians. Bone Joint J 2013;95-B(02):279-285

15 Güler O, Şeker A, Mutlu S, Çerçi MH, Kömür B, Mahiroğulları M Results of a universal ultrasonographic hip screening program at a single institution. Acta Orthop Traumatol Turc 2016;50(01): $42-48$

16 El Jashi R, Gustafson MB, Jakobsen MB, et al. The association between gender and familial prevalence of hip dysplasia in Danish patients. Hip Int 2017;27(03):299-304

17 Azzopardi T, Van Essen P, Cundy PJ, Tucker G, Chan A. Late diagnosis of developmental dysplasia of the hip: an analysis of risk factors. J Pediatr Orthop B 2011;20(01):1-7

18 Lehmann HP, Hinton R, Morello P, Santoli J. Developmental dysplasia of the hip practice guideline: technical report. Committee on Quality Improvement, and Subcommittee on Developmental Dysplasia of the Hip. Pediatrics 2000;105(04):E57

19 Atalar H, Sayli U, Yavuz OY, Uraş I, Dogruel H. Indicators of successful use of the Pavlik harness in infants with developmental dysplasia of the hip. Int Orthop 2007;31(02):145-150

20 Carlile GS, Woodacre T, Cox PJ. Verification of hip reduction using anterior ultrasound scanning during Pavlik harness treatment of developmental dysplasia of the hip. J Orthop 2014;11(04): 174-179

21 Kyung BS, Lee SH, Jeong WK, Park SY. Disparity between Clinical and Ultrasound Examinations in Neonatal Hip Screening. Clin Orthop Surg 2016;8(02):203-209 